ARTICLE

\title{
Real-space observation of incommensurate spin density wave and coexisting charge density wave on $\mathrm{Cr}$ (001) surface
}

Yining Hu (1) 1,5, Tianzhen Zhang ${ }^{1,5}$, Dongming Zhao ${ }^{1,5}$, Chen Chen ${ }^{1}$, Shuyue Ding ${ }^{1}$, Wentao Yang ${ }^{1}, X^{\prime}$ Wang $^{1}$, Chihao Li ${ }^{1}$, Haitao Wang ${ }^{1}$, Donglai Feng (1) ${ }^{2,3,4}$ \& Tong Zhang (1) 1,3,4凶

In itinerant magnetic systems, a spin density wave (SDW) state can be induced by Fermi surface nesting and electron-electron interaction. It may intertwine with other orders such as charge density wave (CDW), while their relation is still yet to be understood. Here via spinpolarized scanning tunneling microscopy, we directly observed long-range spin modulation on $\mathrm{Cr}(001)$ surface, which corresponds to the well-known incommensurate SDW of bulk $\mathrm{Cr}$. It displays $6.0 \mathrm{~nm}$ in-plane period and anti-phase behavior between adjacent (001) planes. Meanwhile, we simultaneously observed the coexisting CDW with half the period of SDW. Such SDW/CDW have highly correlated domain structures and are in-phase. Surprisingly, the CDW displays a contrast inversion around a density-of-states dip at $-22 \mathrm{meV}$, indicating an anomalous CDW gap opened below $E_{\mathrm{F}}$. These observations support that the CDW is a secondary order driven by SDW. Our work is not only a real-space characterization of incommensurate SDW, but also provides insights on how SDW and CDW coexist.

\footnotetext{
${ }^{1}$ State Key Laboratory of Surface Physics, Department of Physics, and Advanced Materials Laboratory, Fudan University, 200438 Shanghai, China. ${ }^{2}$ Hefei National Laboratory for Physical Science at Microscale and Department of Physics, University of Science and Technology of China, 230026 Hefei, Anhui, China. ${ }^{3}$ Collaborative Innovation Center of Advanced Microstructures, 210093 Nanjing, China. ${ }^{4}$ Shanghai Research Center for Quantum Sciences, 201315 Shanghai, China. ${ }^{5}$ These authors contributed equally: Yining Hu, Tianzhen Zhang, Dongming Zhao. ${ }^{凶}$ email: tzhang18@fudan.edu.cn
} 
spin density wave (SDW) state manifests itself as realspace spin modulations. It is usually formed in itinerant magnetic systems with Fermi surface nesting and electron-electron interactions ${ }^{1}$. The spatial period of SDW could be commensurate (C-SDW) or incommensurate (IC-SDW) to lattice constant. In the latter case, the spin modulation decouples from lattice, which is distinguished from local moment induced anti-ferromagnetic (AFM) order. Interestingly, SDW often coexists and sometimes intertwines with other orders in correlated systems, such as charge density wave (CDW) and superconductivity ${ }^{1-4}$. The interplay of these coexisting/intertwining orders has now become an important theme in condensed matter physics. To date, the commonly observed SDW states are commensurate SDW, such as the collinear/bicollinear SDW (AFM) state in iron-based superconductors ${ }^{4,5}$; while incommensurate SDW is rarely seen, and particularly, its real space imaging is quite lacking.

Chromium $(\mathrm{Cr})$ is one of the classic examples which shows itinerant magnetism with an IC-SDW ground state $e^{6-8}$ below its Néel temperature $\left(T_{\mathrm{N}}=311 \mathrm{~K}\right)$. Such IC-SDW is stabilized by "imperfect" Fermi surface nesting condition", as illustrated in Fig. 1a. Specifically, the Fermi surfaces of Cr (b.c.c. lattice) are composed of hole pockets at the corner and electron pocket at the center of the Brillouin zone ${ }^{6}$. The hole pocket is slightly larger than the electron pocket which yields two nesting vectors: $\mathbf{Q}_{ \pm}=2 \pi / a(1 \pm \delta)(a=2.9 \AA$ being the lattice constant $)$. Therefore, a long period IC-SDW with a wave vector $\mathbf{Q}_{\mathrm{SDW}}=2 \pi \delta / a$ is generated which overlaps with the AFM coupling between $\mathrm{Cr}$ atoms (Fig. 1b). The wavelength of IC-SDW is reported to be $6.0 \mathrm{~nm}$ at $T<10 \mathrm{~K}$ (ref. ${ }^{10}$ ), and $\mathbf{Q}_{\mathrm{SDW}}$ is along one of the $<001>$ directions. The spin orientation of $\mathrm{Cr}$ atom is found to be perpendicular to $\mathbf{Q}_{\mathrm{SDW}}$ at $T>T_{\mathrm{SF}}(123 \mathrm{~K})$ but switched to be parallel at $T<T_{\mathrm{SF}}$ (spin-flip transition ${ }^{6}$ ).
In addition to IC-SDW, a charge density wave (CDW) with half period of the IC-SDW was also found in $\mathrm{Cr}^{10-13}$. Unlike the IC-SDW, the exact origin of such CDW is yet to be understood. It was often considered as the second-order harmonics of IC$\mathrm{SDW}^{12}$, corresponding to a nesting vector $\mathbf{Q}_{\mathrm{CDW}}=2 \mathbf{Q}_{\mathrm{SDW}}$ that connects the two folded bands at $\Gamma$ (Fig. 1a); alternatively, it was suggested as a lattice strain wave induced by magneto-elastic coupling to the IC-SDW ${ }^{6}$. Therefore, being a pure element arranged in a simple structure, $\mathrm{Cr}$ is also a classical system to study the interplay of SDW/CDW orders.

However, after decades of research, the characterization of ICSDW (and CDW) in Cr is still rather limited to spatially averaged method, such as neutron scattering ${ }^{6,7}, \mathrm{x}$-ray diffraction ${ }^{10,13}$ and photoemission spectroscopy ${ }^{14,15}$. In principle, SDW could also be detected by local probes at atomic scale, such as spin-polarized scanning tunneling microscopy (SP-STM $)^{16}$. Although a few SPSTM studies have been performed on various $\mathrm{Cr}$ surfaces ${ }^{17-26}$, the real-space evidence of IC-SDW was rarely reported (some studies found CDW modulation on $\mathrm{Cr}$ (110) surface ${ }^{23,27}$, and argued the satellite FFT spots as an indication of IC-SDW ${ }^{23}$ ). Most SP-STM studies on Cr (001) surface only observed in-plane ferromagnetism with AFM coupling between adjacent (001) planes $^{17-22}$. To understand such a ferromagnetic spin arrangement on surface, it was suggested the magnetic moment is enhanced at the surface ${ }^{28}$ and the IC-SDW antinodes are always pinned on the surface ${ }^{8,19}$, making it invisible to STM. However, we noticed that most previous STM studies on $\mathrm{Cr}(001)$ did not resolve clear atomic lattices, although mono-atomic terrace can be identified. This is likely due to local disorders induced by segregated impurities on the surface, which is a common problem in cleaning $\mathrm{Cr}$ single crystal. As the surface conditions could alter the surface magnetism ${ }^{8}$, it would be intriguing to search the IC$\mathrm{SDW}$ in real space again on a well-ordered $\mathrm{Cr}$ surface.
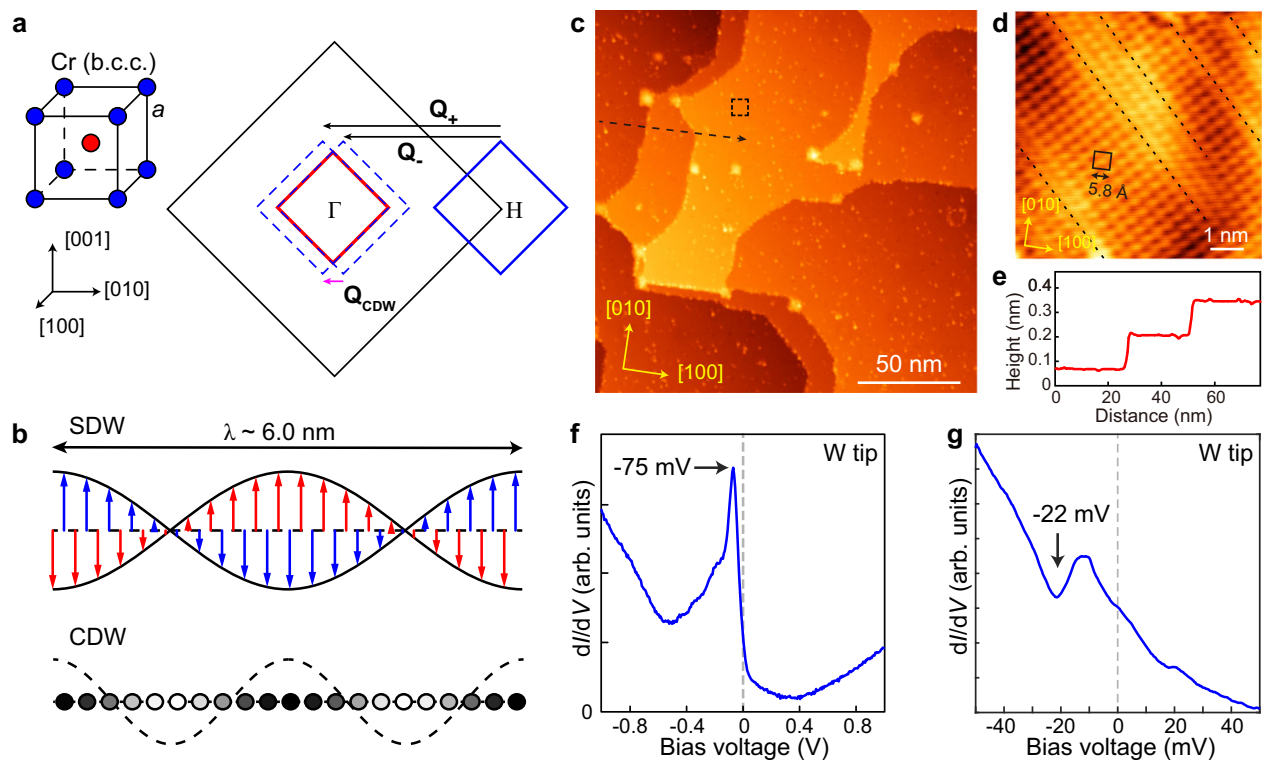

Fig. 1 Sketch of the crystal structure and SDW/CDW states of bulk $\mathbf{C r}$, and STM characterization of $\mathbf{C r ( 0 0 1 )}$ surface. a Left: the body-centered cubic (b.c.c.) structure, and right: the (001) plane Brillouin zone of $\mathrm{Cr}$. Electron and hole Fermi surfaces (cross sections) are represented by red and blue squares, respectively. The nesting vectors $\mathbf{Q}_{ \pm}=2 \pi / a(1 \pm \delta)$ are indicated by black arrows, and $\mathbf{Q}_{\mathrm{CDW}}=\mathbf{Q}_{+}-\mathbf{Q}_{-}$is the CDW wave vector. $\mathbf{b}$ Schematic of incommensurate SDW and CDW of bulk $\mathrm{Cr}$ in real space. Blue and red arrows represent the spin of corner and body-center $\mathrm{Cr}$ atoms in the b.c.c. lattice at $T>T_{\mathrm{SF}}$, respectively. Solid (hollow) circles represent the locations with the highest (lowest) charge density. c Large scale STM image (190 $\left.\times 190 \mathrm{~nm}{ }^{2}\right)$ of the cleaned $\mathrm{Cr}$ (001) surface. d Atomically resolved STM image (taken in the dashed square in panel c) showing a centered ( $2 \times 2)$ structure. The dashed lines indicate the surface line-dislocations (see Supplementary Fig. S1 for more details). e Line profile taken along the dashed line in panel (c). $\mathbf{f}$ Typical dl/ $\mathrm{d} V$ point spectrum taken on a defect-free region with a normal $W$ tip (setpoint: $V_{b}=1 \mathrm{~V}, I=60 \mathrm{pA}, \Delta V=20 \mathrm{mV}$ ). $\mathbf{g}$ Typical $\mathrm{d} l / \mathrm{d} V$ spectrum taken around $E_{\mathrm{F}}$ by $\mathrm{W}$ tip (setpoint: $V_{\mathrm{b}}=50 \mathrm{mV}, I=100 \mathrm{pA}, \Delta V=1 \mathrm{mV}$ ), a DOS dip at $V_{\mathrm{b}} \approx-22( \pm 1) \mathrm{mV}$ is observed. 
In this work, by using low-temperature spin-polarized STM with vector magnetic field, we studied a thoroughly cleaned $\mathrm{Cr}$ (001) with a well-ordered surface. We observed clear spin modulation with a period of $6.0 \mathrm{~nm}$, propagating along in-plane [100] or [010] directions, which well matches the projected bulk ICSDW on (001) surface. Its SDW nature is confirmed by the contrast inversion upon switching tip's magnetization, and the anti-phase relation between adjacent terraces. Meanwhile, we also observed the coexisting CDW with a period $3.0 \mathrm{~nm}$, and surprisingly found that it displays a $\pi$ phase shift around gap structure about $22 \mathrm{meV}$ below $E_{\mathrm{F}}$, which suggests its formation is beyond the intuitive Fermi surface nesting picture. Furthermore, as a local probe measurement, we directly observed the domain structure of SDW/CDW and revealed their in-phase relation. Our work not only gives a real-space investigation of IC-SDW, but also provide new insights on the general mechanism of coexisting SDW/CDW orders.

\section{Results}

STM characterization of Cr (001) surface and it's tunneling spectrum. The experiment was conducted in a cryogenic STM (UNISOKU) at $T=5.0 \mathrm{~K}$. Details about the cleaning process of $\mathrm{Cr}$ (001) and STM measurement are described in the "Methods" section. Figure 1c shows a large scale STM image of the obtained $\mathrm{Cr}$ (001) surface. It displays atomically flat terraces with monoatomic height $(\approx 0.14 \mathrm{~nm})$, as indicated by the line profile in Fig. 1e. It is notable that the terrace edges prefer running along high symmetric directions such as [100], [010], and [110]. This is an indication of free surface atom diffusion during annealing ${ }^{29}$. Despite some randomly distributed defects, atomic lattice can be easily resolved in defect free area, as shown in Fig. 1d. It displays a centered $2 \times 2$ (or $\left.\sqrt{2} \times \sqrt{2} \mathrm{R}_{4} 5^{\circ}\right)$ lattice with respect to the pristine $\mathrm{Cr} 1 \times 1$ lattice. Some dislocation lines are observed where the atomic lattice on their two sides displays certain shift (black dashed lines in Fig. 1d). These dislocations do not show influence to SDW/CDW discussed below, more details are presented in Supplementary Fig. S1. We noticed some previous STM works on $\mathrm{Cr}(001)$ also observed $c(2 \times 2)$ structures $22,26,30$, but the electronic states of the present surface is quite different from those studies (shown below). Although the origin of this reconstruction is unclear at this stage, it is the first time to observe regular oriented terrace edges with well-ordered lattice on a sputtered/annealed $\mathrm{Cr}$ (001) surface.

The typical large energy scale $\mathrm{d} I / \mathrm{d} V$ spectrum of the surface, measured by a normal $\mathrm{W}$ tip above defect-free area, is shown in Fig. 1f. There is a pronounced DOS peak located at $-75( \pm 5) \mathrm{mV}$. We note although a DOS peak was widely observed on $\mathrm{Cr}$ surfaces $^{18-26,31,32}$, the peak position varies significantly for different studies. The origin of such a peak was usually attributed to spin-polarized surface state st,33-35 $^{3}$ or the orbital Kondo effect $^{32}$. Our measurements shown below tend to support the former scenario. By zooming into a narrower energy range near $E_{\mathrm{F}}$ (Fig. 1g), we found there is an additional DOS dip at $E=-22( \pm 1) \mathrm{meV}$, which has never been reported before. Such a DOS dip is repeatedly observed at different surface locations (see Supplementary Fig. S2 for more spectra). We will show later that it is likely an energy gap associated with the CDW order, but opens below the Fermi level.

Real-space imaging of incommensurate SDW. We then studied the surface with spin-polarized tips. Fig. 2a is a $d I / d V$ map taken at $V_{\mathrm{b}}=-150 \mathrm{meV}$ with a tip coated with $40 \mathrm{~nm}$ thick $\mathrm{Cr}$, which favors an in-plane spin polarization ${ }^{16}$. The mapping area is the same as that shown Fig. 1c. It is remarkable that stripe-like modulations can be observed, and there are two domains of such modulation which are perpendicular to each other. A zoomed-in $\mathrm{d} I / \mathrm{d} V$ map around a domain wall is shown in Fig. $2 \mathrm{~b}$. The period of the stripe is $6.0 \mathrm{~nm}$ and the wave vector is either along [010] or [100] direction (as also seen in the FFT image in Fig. 2a inset). Such a period and propagating direction exactly match the projected bulk IC-SDW of Cr on a (001) surface. It is also seen that the domain walls in Fig. 2a (dashed curve) have no correlation with surface morphology (Fig. 1c), which indicates the stripes are not merely surface effects but of bulk origin. Figure $2 \mathrm{c}$ shows the typical $\mathrm{d} I / \mathrm{d} V$ spectra taken on the stripe and between the stripes with the Cr-coated tip. There is observable difference on the intensity around the DOS peak, which is attributed to spin contrast as discussed below. More $\mathrm{d} I / \mathrm{d} V$ maps taken at different energies and their FFT images can be found in Supplementary Figs. S3 and S4.

To further verify these stripes are spin modulations, we performed measurement with a $16 \mathrm{~nm}$-Fe-coated tip whose magnetization can be controlled by an external magnetic field ${ }^{16}$. Figure $3 \mathrm{a}, \mathrm{b}$ are two $\mathrm{d} I / \mathrm{d} V$ maps taken in the same region, but under opposite in-plane field of $B_{\mathrm{X}}= \pm 1 \mathrm{~T}$ ( $\mathrm{X}$ direction is perpendicular to stripes, as marked in figure). The $6.0 \mathrm{~nm}$ period stripes can be seen in both Fig. 3a, b, while they display a clear phase inversion, as further illustrated in their line profiles in Fig. 3e. Since the tip magnetization will follow such in-plane field, this gives a direct evidence that the stripes are SDW modulations, with opposite spin orientations on their peaks and troughs. We can also tune the tip magnetization along $\mathbf{Y}$ and $\mathbf{Z}$ directions (by applying $B_{\mathrm{Y}}=1 \mathrm{~T}$ and $B_{\mathrm{Z}}=1.5 \mathrm{~T}$, respectively), the resulting $\mathrm{d} I /$ $\mathrm{d} V$ maps are shown in Fig. $3 \mathrm{c}, \mathrm{d}$, respectively. In these two cases, the contrast of the stripes was significantly reduced and almost invisible. This confirmed that the spins are only polarized along $\mathbf{X}$ direction (the same direction of $\mathbf{Q}_{\mathrm{SDW}}$ ), which agrees with bulk measurements that the IC-SDW is longitudinal wave at $T<T_{\mathrm{SF}}$ (ref. ${ }^{6}$ ). We can extract the spin-polarization ratio (spin-contrast) of Fig. 3a, b by calculating their relative intensity difference, which is about $4 \%$ at the SDW peaks (Fig. 3f). We note here that for Cr-coated tip used in Fig. 2, its (in-plane) polarization direction is arbitrary ${ }^{16}$, that is why the two SDW domains in Fig. $2 \mathrm{a}, \mathrm{b}$ can be simultaneously imaged but their contrasts are different.

We note that the above measurement under vector magnetic field also distinguished the SDW state from "spin-spiral" order which has been detected by SP-STM in other magnetic systems ${ }^{36,37}$. In a spin-spiral, the spins have a nearly constant magnitude but their orientations keep rotating with certain chirality, thus one would observe spin modulation in at least two of the $X, Y, Z$ components (depends on the types of spin-spiral, e.g., helical or cycloidal ${ }^{36}$ ). However here we only observed spin modulation along $\mathrm{X}$ direction.

Another signature of IC-SDW can be obtained near the atomic step edges. Figure $3 \mathrm{~g}$ shows a topographic image of three adjacent mono-atomic height terraces, and Fig. $3 \mathrm{~h}$ is the corresponding $\mathrm{dI} / \mathrm{dV}$ map measured by a Cr-coated tip. As shown by dashed lines, there is a phase inversion of the modulations between adjacent terraces. This indicates the local spin of two adjacent (001) plane are still AFM coupled. Based on above observations, we now achieve a complete spin configuration of the present $\mathrm{Cr}$ (001) surface. As illustrated in Fig. 3i, the $\mathrm{Cr}$ have an out-of-plane AFM configuration with the spins lying in-plane, while a long wavelength, longitudinal IC-SDW $(\lambda=6.0 \mathrm{~nm})$ is present in each (001) planes. Such a magnetic structure agrees with the neutron-scattering measurement for bulk $\mathrm{Cr}$ and thick $\mathrm{Cr}$ films ${ }^{6-8}$, but has not been visualized by a local probe before. Comparing with the commensurate SDW or AFM state ${ }^{4,16}$, the IC-SDW observed here are pure spin modulations that decoupled from the lattice. It can be considered as a "modulated" ferromagnetism for the top Cr plane. 

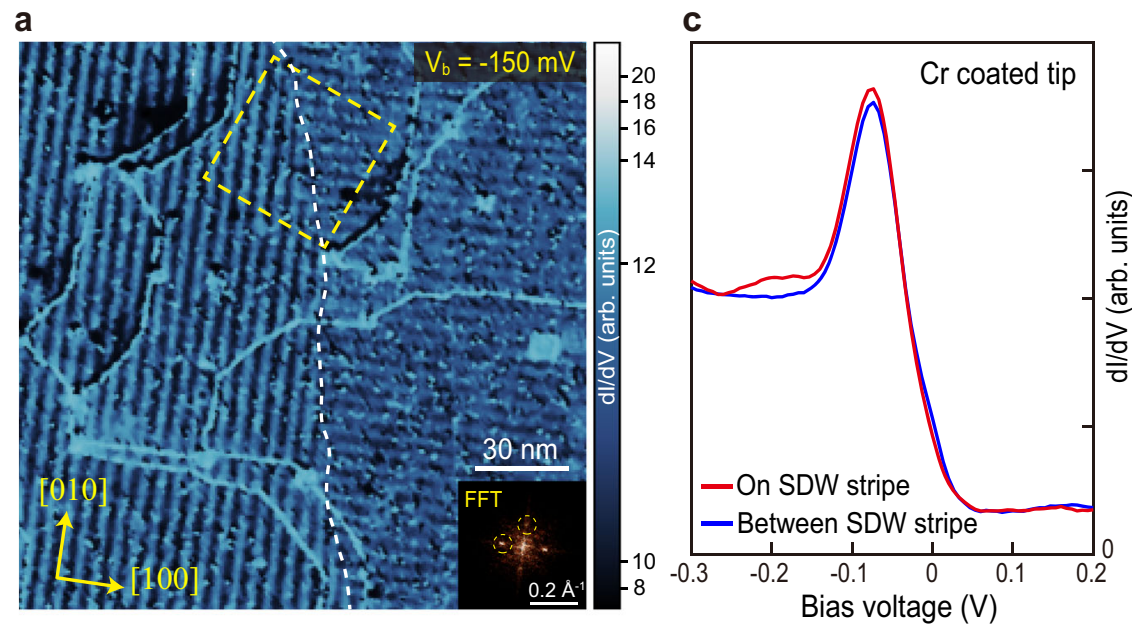

b

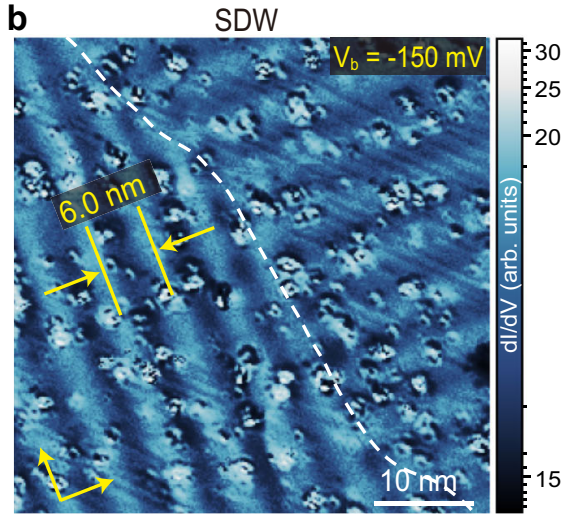

d

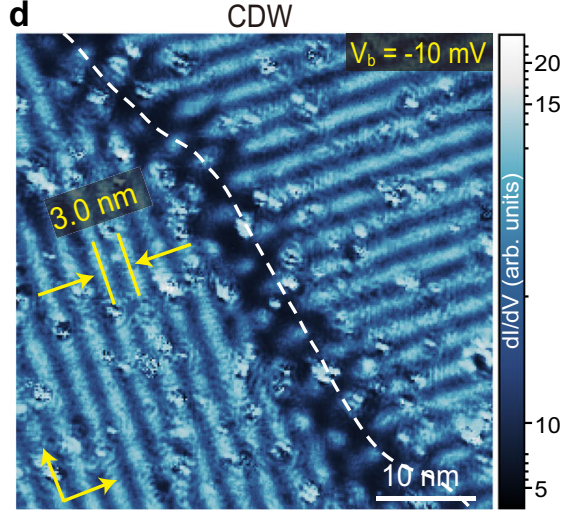

Fig. 2 Spin and charge modulations observed on $\mathbf{C r}$ (001) surface. a The $\mathrm{d} / \mathrm{d} V$ map of the same area that shown in Fig. $1 \mathrm{c}$, taken by a $\mathrm{Cr}$ coated spinpolarized tip at $V_{b}=-150 \mathrm{mV}(I=80 \mathrm{pA}, \Delta V=20 \mathrm{mV})$. Inset: FFT image, dashed circles indicate the spots from spin modulations. $\mathbf{b} \mathrm{A}$ zoom-in $\mathrm{dl} / \mathrm{dV}$ map of the marked square in $\mathbf{a}$, which shows spin modulation with a period of $6.0 \mathrm{~nm}$. White dashed curve tracks the domain wall $\left(V_{\mathrm{b}}=-150 \mathrm{mV}, I=150\right.$ $\mathrm{pA}, \Delta V=15 \mathrm{mV}$ ). c Typical $\mathrm{d} / \mathrm{dV}$ (point spectra) taken on and in between the spin modulation stripes (setpoint: $V_{\mathrm{b}}=1 \mathrm{~V}, I=60 \mathrm{pA}, \Delta V=20 \mathrm{mV}$ ). $\mathbf{d} \mathrm{d} l / \mathrm{d} V$ map of the same area as $\mathbf{b}$, but taken at $V_{\mathrm{b}}=-10 \mathrm{mV}$. The charge modulation with a period of $3.0 \mathrm{~nm}$ is observed $(I=150 \mathrm{pA}, \Delta V=5 \mathrm{mV})$.

Observation of the coexisting CDW. Moreover, in addition to the SDW modulation, we also observed another type of modulation with half the period of SDW $(3.0 \mathrm{~nm})$, as shown in the $\mathrm{d} I /$ $\mathrm{d} V$ map in Fig. $2 \mathrm{~d}$ (taken at $V_{\mathrm{b}}=-10 \mathrm{mV}$ ). It displays the same domain structures with the SDW shown in Fig. 2b, however here the two domains have the same contrast. We further verified that such $3.0 \mathrm{~nm}$ modulation is also visible under a nonmagnetic PtIr tip, but the SDW is invisible (see Supplementary Fig. S5). Therefore, it is natural to assign such spin-unpolarized modulation to $\mathrm{CDW}$ with a $\mathbf{Q}_{\mathrm{CDW}}=2 \mathbf{Q}_{\mathrm{SDW}}$, as reported in X-ray studies of $\mathrm{Cr}^{10,13}$. We noticed a previous STM study on $\mathrm{Cr}$ (110) surface reported similar charge modulation that originated from bulk $\mathrm{CDW}^{27}$. Here, we are able to image the SDW and CDW simultaneously, enabling the study of their microscopic correlations.

Figure $4 \mathrm{a}-\mathrm{h}$ show a series of $\mathrm{d} I / \mathrm{d} V$ maps taken in the same region, with the same $\mathrm{Cr}$ tip but at various $V_{\mathrm{b}}$. Their (averaged) line profiles are summarized in Fig. 4j, which display the evolvement of SDW/CDW modulations as the energy varies. Figure $4 \mathrm{i}$ shows the typical $\mathrm{d} I / \mathrm{d} V$ spectrum of this mapping region, and the energy positions corresponding to line profiles in Fig. $4 \mathrm{j}$ are indicated by dashed lines. The SDW modulation is mainly observable in the energy range of $-200 \mathrm{meV} \sim-50 \mathrm{meV}$, which covers the large DOS peak in $\mathrm{d} I / \mathrm{d} V$. This suggests the peak is from certain spin-polarized state(s). As the mapping energy lowered to $-50 \mathrm{meV} \sim 0$, the $3.0 \mathrm{~nm} \mathrm{CDW}$ modulation became pronounced. Interestingly, it displays an abrupt phase inversion between -30 and $-10 \mathrm{meV}$ (see also the $\mathrm{d} I / \mathrm{d} V$ maps in Fig. $4 \mathrm{f}$, g). Such $\pi$ phase shift can also be seen in the phase of the Fourier transformations, as shown in Fig. $4 \mathrm{k}$. We note this energy range right covers the DOS dip in the $\mathrm{d} I / \mathrm{d} V$ spectrum (Fig. $4 \mathrm{i}$ ), which suggests such a DOS dip is from a CDW gap, as the phase inversion of particle-hole states around the gap is a hallmark of $\mathrm{CDW}^{38,39}$. Our measurement at elevated temperatures (shown in Supplementary Figs. S6 and S7) also suggested the DOS dip and CDW are correlated, as they are still both visible at $T=78 \mathrm{~K}$ but disappeared together at $T=301 \mathrm{~K}$, which is close to $T_{\mathrm{N}}=311 \mathrm{~K}$.

However, it is unusual that the gap is not opened at $E_{\mathrm{F}}$ here, but about $22 \mathrm{meV}$ below, which is rather unexpected for conventional $\mathrm{CDW}^{1}$. This gap is also unlikely associated with the SDW as its size $(\approx 10 \mathrm{meV})$ is too small with comparing to the Néel temperature of $\mathrm{Cr}(311 \mathrm{~K})$. We note in previous ARPES study on $\mathrm{Cr}$ (110), a SDW gap is found to be about $200 \mathrm{meV}$ and located above $E_{F}$ (ref. ${ }^{14}$ ), which can be understood through AFM coupling induced band folding (see Supplementary Fig. S8, the hole pocket of $\mathrm{Cr}$ is slightly larger than electron pocket, their crossing point upon folding is above $E_{\mathrm{F}}$ ). However, here we did not observe an obvious SDW gap in tunneling spectrum, although the tunneling conductance at positive energy is indeed low in Fig. 1f (whether it is related to SDW gap needs further investigation). Assuming the $\mathrm{Cr}$ sample here has similar band structure to that reported in ref. ${ }^{14}$, a CDW gap below $E_{\mathrm{F}}$ cannot be induced by the band folding scenario either. It therefore suggests the formation of $\mathrm{CDW}$ is beyond the intuitive Fermi surface nesting picture. We note a recent STM study on $\mathrm{TiSe}_{2}$ also 
a

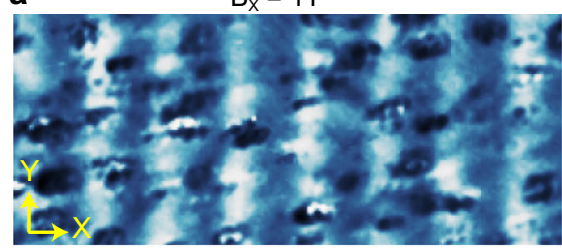

b

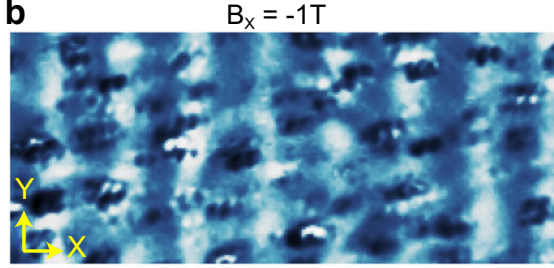

e

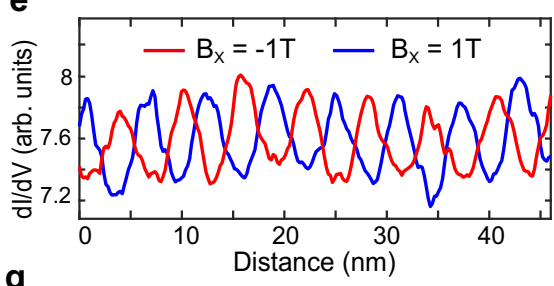

g

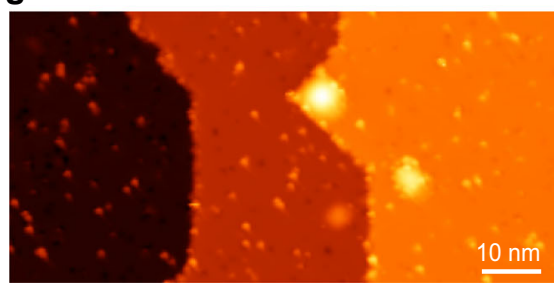

i

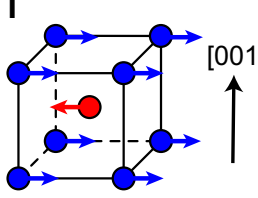

C

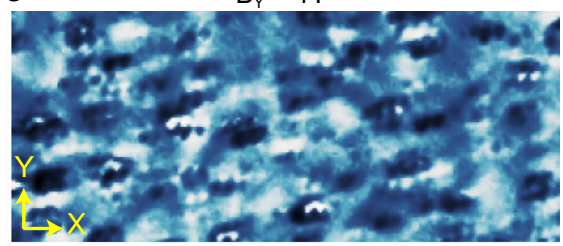

d $\quad B_{z}=1.5 T$

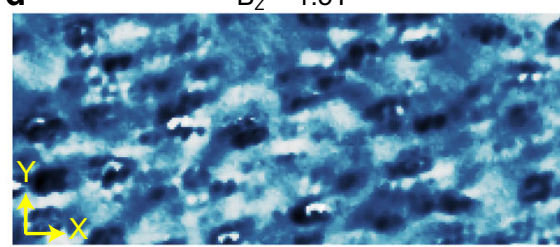

$f$
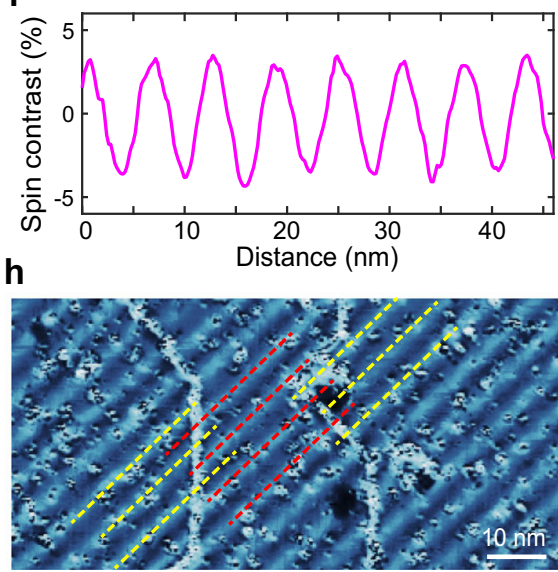

$\operatorname{SDW}(\lambda \sim 6.0 \mathrm{~nm})$

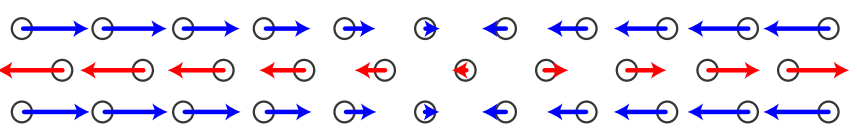

Fig. 3 Verifying the nature of spin density wave. a-d $d / / d V$ maps taken with a Fe-coated tip under different magnet field (marked above each panel). The mapping area is the same for these panels (size: $46 \times 20 \mathrm{~nm}^{2}, V_{b}=-100 \mathrm{mV}, I=80 \mathrm{pA}, \Delta \mathrm{V}=10 \mathrm{mV}$ ). e averaged line profile (along X direction) of $\mathbf{a}$, b. A phase inversion can be clearly seen for $B_{x}= \pm 1 \mathrm{~T}$. $\mathbf{f}$ Spin contrast (polarization ratio) calculated by the relative difference of the line profiles in $\mathbf{e} . \mathbf{g}$ STM image of three adjacent mono-atomic steps. The step height is $\approx 1.45 \AA$. $\mathbf{h} \mathrm{d} l / \mathrm{d} V$ map taken in the same area of panel $\mathbf{g}$, the SDW displays phase inversion across each step edge, as indicated by yellow and red dashed lines $\left(V_{b}=-200 \mathrm{mV}, I=100 \mathrm{pA}, \Delta V=15 \mathrm{mV}\right)$. i A sketch of local spin configuration and ICSDW near $\mathrm{Cr}$ (001) surface.

indicated a CDW gap opened away from $E_{\mathrm{F}}$ (ref. ${ }^{40}$ ), which was attributed to strong electron correlations.

Further information can be extracted from the real space imaging of SDW and CDW is their phase relation. As discussed before, the SDW modulations in $\mathrm{d} I / \mathrm{d} V$ are directly induced by spin contrast (Fig. 3). Their maximum and minimum positions are where the absolute spin density reach maximum. Meanwhile, the CDW modulation in $\mathrm{d} I / \mathrm{d} V$ is the local DOS variation induced by periodic charge distribution ${ }^{38}$. Figure $4 j$ shows that the locations with maximum spin density (tracked by solid and dashed lines) always have minimum LDOS at $-30 \mathrm{mV}$ and maximum LDOS at $-10 \mathrm{meV}$. As usually the charge density is proportional to the LDOS of occupied state near $E_{\mathrm{F}}$, our data suggests the SDW and CDW in $\mathrm{Cr}$ are in-phase, i.e., the positions with maximum spin density also have maximum charge density (sketched in Fig. 1b). We note that previously such relation can only be obtained through combined X-ray and neutron diffraction measurement after extensive data analysis ${ }^{41,42}$, while here we provide a rather direct evidence on the same system. The in-phase relation appears to consist with the theory which treat $\mathrm{CDW}$ as a second harmonics of SDW ${ }^{12,43,44}$.

At last, beside the static SDW/CDW modulations, we also observed dispersive quasi-particle interference (QPI) on the surface. As shown in Fig. 5a for example, clear short wavelength interference patterns are visible around the defects. Figure $5 \mathrm{~b}$ is the FFT image of Fig. 5a which displays a square shaped scattering ring (more QPI data is shown in Supplementary Fig. S9). By summarizing the FFT line profile taken at various energies (Fig. 5c), an electron-like dispersion with $q_{\mathrm{F}} \approx 1.1 \AA^{-1}$ is visualized. We note previous DFT calculations had predicted multiple spin-polarized surface states on $\mathrm{Cr}(001)^{33}$, the observed QPI could be originated from one of the surface states, as bulk bands usually do not generate strong QPI; and the DOS peak at $E=-75 \mathrm{meV}$ in $\mathrm{d} I / \mathrm{d} V$ (Fig. 1f) could be from the onset of this band. The clear observation of QPI here (which is absent in previous STM studies) is also an indication of improved surface condition. We expect it will help to elucidate the surface electronic states of $\mathrm{Cr}$ with the help of further theoretical calculations. 

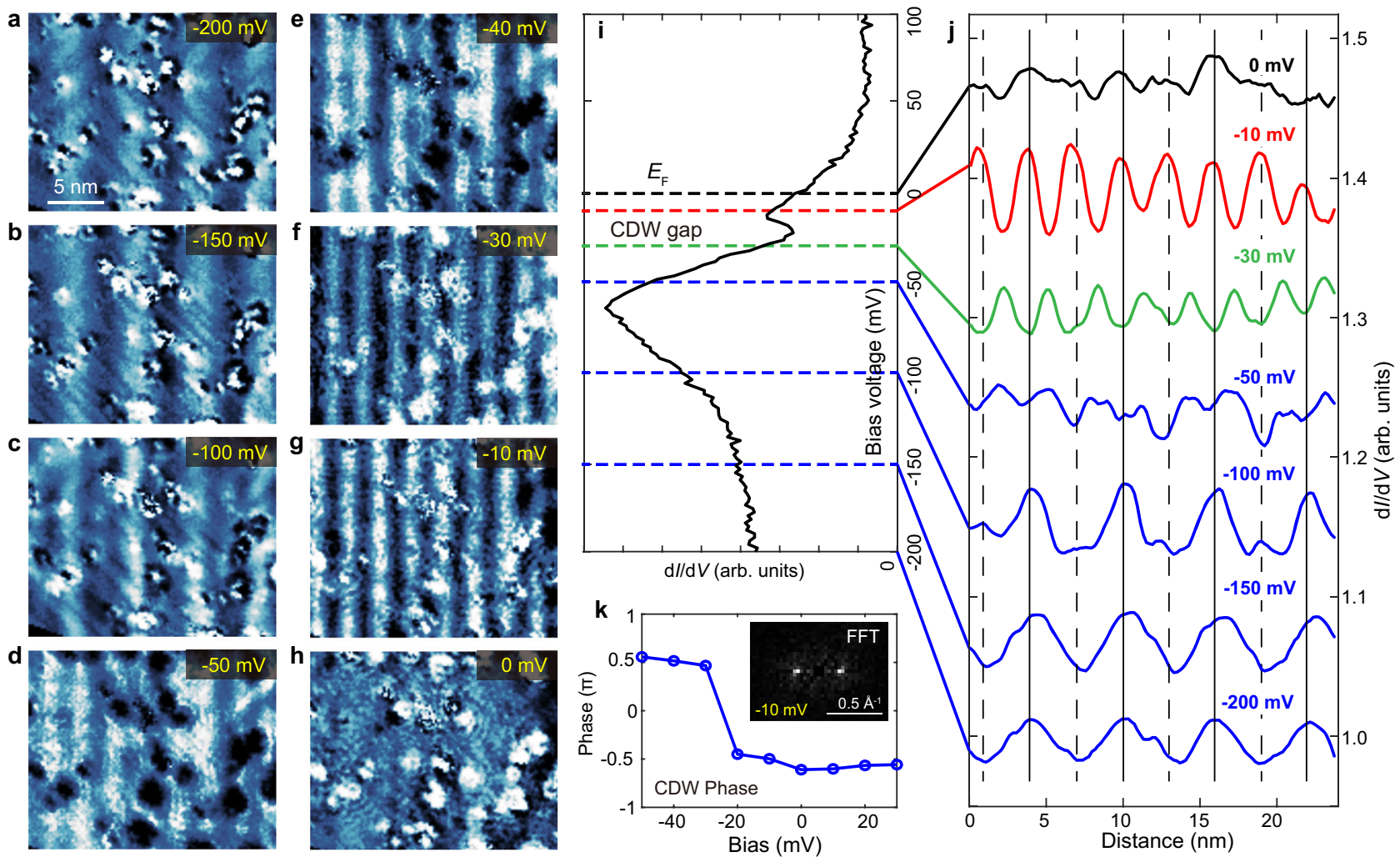

Fig. 4 Evolvement of SDW/CDW modulations with energy, and the phase inversion of CDW. a-h $\mathrm{d} / / \mathrm{d} V$ maps taken by $\mathrm{Cr}$-coated tip at various $V_{\mathrm{b}}$. The mapping area are the same for these panels. $\mathbf{i}$ The typical $\mathrm{d} l / \mathrm{d} V$ point spectrum taken in this region (setpoint: $V_{\mathrm{b}}=-500 \mathrm{mV}, I=60 \mathrm{pA}, \Delta V=10 \mathrm{mV}$ ). j Averaged line profile of the $\mathrm{d} / \mathrm{d} V \mathrm{~V}$ maps. An abrupt phase inversion can be seen between $V_{\mathrm{b}}=-30$ and $-10 \mathrm{mV}$, which corresponds to the $\mathrm{DOS}$ dip region in $\mathbf{i}$. $\mathbf{k}$ The phase of the CDW modulation, which are extracted from the raw FFT values at $\mathbf{Q}_{\mathrm{CDW}}$. Inset image shows the FFT of $\mathbf{g}\left(V_{\mathrm{b}}=-10 \mathrm{mV}\right)$ which displays $\mathbf{Q}_{\mathrm{CDW}}$ spots at $\pm 2 \pi / \lambda_{\mathrm{CDW}}$.
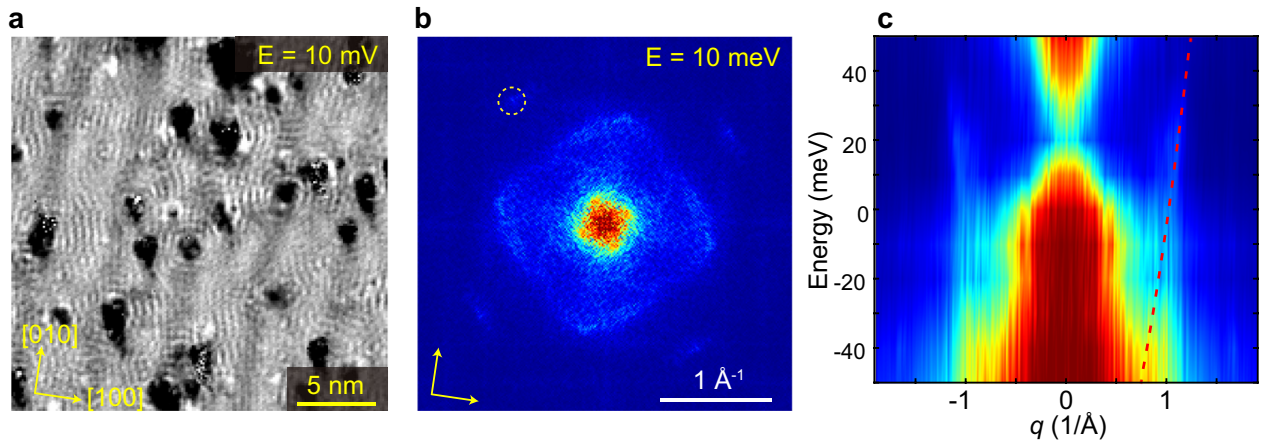

Fig. 5 QPI measurement on $\mathbf{C r}$ (001) surface. a $d / / d V$ image taken at $V_{b}=10 \mathrm{mV}(I=100 \mathrm{pA})$, which shows the QPI modulation. b The four-fold symmetrized FFT image of at $E=10 \mathrm{meV}$. The dashed circle indicated the FFT spots from $\mathrm{c}(2 \times 2)$ reconstruction. $\mathbf{c}$ Color plot of the summarized FFT line cuts, dashed line tracks the dispersion of electron-like band.

\section{Discussion}

We have now presented a comprehensive SP-STM study on a wellordered $\mathrm{Cr}$ (001) surface. We directly observed the incommensurate SDW with similar spin configurations to the bulk, which manifests as long-period, linearly polarized pure spin modulations on the surface; and the coexisting CDW order is also simultaneously observed. These features are absent in previous STM studies which indicates the surface condition is important for probing intrinsic magnetism of $\mathrm{Cr}$. Another main finding of this work is the CDW gap opened below $E_{\mathrm{F}}$. It appears conflict with conventional Fermi surface nesting picture in which the density wave gap will open at $E_{\mathrm{F}}$ to lower the energy. Nonetheless, if considering the CDW in $\mathrm{Cr}$ is driven by SDW ${ }^{12,43,44}$, the system would gain energy mainly through SDW and it is possible the formation of CDW involves states away from $E_{\mathrm{F}}$. This implies additional factors, such as electron correlations, may play a role in $\mathrm{CDW}$ of $\mathrm{Cr}$. Moreover, as a real space measurement, we can directly observe the domain and phase relations of SDW and CDW. The same domain structure and an in-phase relation of local spin and charge indicate these two orders are highly correlated, consistent with the scenario that CDW is the high-order harmonics of SDW.

The mechanism of coexisting spin/charge orders has long been an important issue in condensed matter physics, particularly for correlated materials such as cuprates ${ }^{3,45}$ and iron-based superconductors ${ }^{4,46}$, our new spectroscopic and microscopic information provide insights on the comprehensive understanding SDW/ $\mathrm{CDW}$ in $\mathrm{Cr}$ and other correlated materials. Our work is one of the 
few cases in which simultaneous imaging of spin/charge order with high resolution is achieved (ref. ${ }^{5}$ is another example). We expected similar SP-STM measurement shall also be applied to other systems and would inspire more studies on the coexisting quantum orders.

\section{Methods}

Cr (001) sample preparation and STM measurements. Cr (001) single crystal (Mateck, purity: 99.999\%) was intensively cleaned by repeated cycles of Ar sputtering at $750^{\circ} \mathrm{C}$ (for $15 \mathrm{~min}$ ) and annealing at $800^{\circ} \mathrm{C}$ (for $20 \mathrm{~min}$ ), until a well-ordered surface is obtained. Spin-resolved tunneling spectroscopy and conductance mapping were performed by Cr-coated and Fe-coated STM tips, which are prepared by depositing $40 \mathrm{~nm}$ $\mathrm{Cr}$ or $16 \mathrm{~nm}$ Fe layers on $\mathrm{W}$ tip. The $\mathrm{W}$ tip was electrochemically etched and flashed up to $\approx 2000 \mathrm{~K}$ for cleaning before coating. The tunneling conductance $(\mathrm{d} I / \mathrm{d} V)$ was collected by standard lock-in method and the bias voltage $\left(V_{\mathrm{b}}\right)$ is applied to the sample.

\section{Data availability}

The main data supporting the findings of this study are available within the article and its Supplementary Information files. All the raw data generated in this study are available from the corresponding author upon reasonable request.

\section{Code availability}

All the data analysis codes related to this study are available from the corresponding author upon reasonable request.

Received: 11 July 2021; Accepted: 10 January 2022;

Published online: 21 January 2022

\section{References}

1. Gruner, G. Density Waves in Solids. (Addison-Wesley Publishing, 1994).

2. Gabovich, A. M. et al. Charge- and spin-density-wave superconductors. Supercond. Sci. Technol. 14, R1 (2001).

3. Kivelson, S. A. et al. How to detect fluctuating stripes in the high-temperature superconductors. Rev. Mod. Phys. 75, 1201 (2003).

4. Dai, P. C., Hu, J. P. \& Dagotto, E. Magnetism and its microscopic origin in iron-based high-temperature superconductors. Nat. Phys. 8, 709 (2012).

5. Enayat, M. et al. Real-space imaging of the atomic-scale magnetic structure of $\mathrm{Fe}_{1+\mathrm{y}} \mathrm{Te}$. Science 345, 653 (2014).

6. Fawcett, E. Spin-density-wave antiferromagnetism in Cr. Rev. Mod. Phys. 60, 209 (1988).

7. Fawcett, E. et al. Spin-density-wave antiferromagnetism in chromium alloys. Rev. Mod. Phys. 66, 25 (1994).

8. Zabel, H. Magnetism of chromium at surfaces, at interfaces and in thin films. J. Phys. Condens. Matter 11, 9303 (1999).

9. Overhauser, A. W. Spin density waves in an electron gas. Phys. Rev. 128, 1437 (1962).

10. Gibbs, D. \& Mohanty, K. M. High-resolution x-ray-scattering study of chargedensity-wave modulation in chromium. Phys. Rev. B 37, 562 (1988).

11. Tsunoda, Y., Mori, M., Kunitomi, N., Teraoka, Y. \& Kanamori, J. Strain wave in pure chromium. Solid State Commun. 14, 287 (1974).

12. Young, C. Y. \& Sokoloff, J. B. The role of harmonics in the first order antiferromagnetic to paramagnetic transition in chromium. J. Phys. F 4, 1304 (1974).

13. Hill, J. P., Helgesen, G. \& Gibbs, D. X-ray-scattering study of charge- and spin-density waves in chromium. Phys. Rev. B 52, 7363 (1995).

14. Schäfer, J., Rotenberg, E., Meigs, G. \& Kevan, S. D. Direct spectroscopic observation of the energy gap formation in the spin density wave phase transition at the $\operatorname{Cr}(110)$ surface. Phys. Rev. Lett. 83, 2069 (1999).

15. Rotenberg, E. et al. Electron states and the spin density wave phase diagram in Cr(110) films. New J. Phys. 7, 114 (2005)

16. Wiesendanger, R. Spin mapping at the nanoscale and atomic scale. Rev. Mod. Phys. 81, 1495 (2009).

17. Wiesendanger, R. et al. Observation of vacuum tunneling of spin-polarized electrons with the scanning tunneling microscope. Phys. Rev. Lett. 65, 247 (1990).

18. Kleiber, M. et al. Topology-induced spin frustrations at the $\mathrm{Cr}(001)$ surface studied by spin-polarized scanning tunneling spectroscopy. Phys. Rev. Lett. 85, 4606 (2000).

19. Hänke, T. et al. Absence of spin-flip transition at the $\operatorname{Cr}(001)$ surface: a combined spin-polarized scanning tunneling microscopy and neutron scattering study. Phys. Rev. B 71, 184407 (2005).

20. Kleiber, M. et al. Magnetic properties of the $\operatorname{Cr}(001)$ surface studied by spinpolarized scanning tunneling spectroscopy. J. Magn. Magn. Mater. 240, 64 (2002).

21. Kawagoe, T. et al. Evidence of a topological antiferromagnetic order on ultrathin $\mathrm{Cr}(001)$ film surface studied by spin-polarized scanning tunneling spectroscopy. J. Appl. Phys. 93, 6575-6577 (2003).
22. Oka, H. \& Sueoka, K. Spin-polarized scanning tunneling microscopy and spectroscopy study of $\mathrm{c}(2 \times 2)$ reconstructed $\mathrm{Cr}(001)$ thin film surfaces. J. Appl. Phys. 99, 08D302 (2006).

23. Santos, B. et al. Structure and magnetism of ultra-thin chromium layers on $\mathrm{W}(110)$. New J. Phys. 10, 013005 (2008).

24. Lagoute, J. et al. Spin-polarized scanning tunneling microscopy and spectroscopy study of chromium on a $\mathrm{Cr}(001)$ surface. J. Phys. Condens. Matter 23, 045007 (2011).

25. Hsu, P. J. et al. Observation of a spin-density wave node on antiferromagnetic Cr(110) islands. Phys. Rev. B 87, 115437 (2013).

26. Corbett, J. P. \& Smith, A. R. Applying a difference ratio method in spinpolarized scanning tunneling microscopy to determine crystalline anisotropies and antiferromagnetic spin alignment in $\operatorname{Cr}(001) \mathrm{c}(2 \times 2)$. J. Magn. Magn. Mater. 465, 626-633 (2018).

27. Braun, K. F. et al. Observation of charge-density wave domains on the $\mathrm{Cr}(110)$ surface by low-temperature scanning tunneling microscopy. Phys. Rev. Lett. 85, 3500 (2000).

28. Allan, G. Surface electronic structure of antiferromagnetic chromium. Surf. Sci. 74, 79 (1978).

29. Jeong, H. C. \& Williams, E. D. Steps on surfaces: experiment and theory. Surf. Sci. Rep. 34, 171-294 (1999).

30. Schmid, M. et al. Segregation of impurities on $\operatorname{Cr}(100)$ studied by AES and STM. Surf. Sci. 377-379, 1023 (1997).

31. Stroscio, J. A. et al. Tunneling spectroscopy of bcc (001) surface states. Phys. Rev. Lett. 75, 2960 (1995).

32. Kolesnychenko, O. Y. et al. Real-space imaging of an orbital Kondo resonance on the $\mathrm{Cr}(001)$ surface. Nature 415, 507 (2002).

33. Habibi, P. et al. Electronic and magnetic structure of the $\mathrm{Cr}(001)$ surface. $J$. Phys. Condens. Matter 25, 146002 (2013).

34. Leroy, M. A. et al. Electronic structure of the $\mathrm{Cr}(001)$ surface and $\mathrm{Cr} / \mathrm{MgO}$ interface. Phys. Rev. B 88, 205134 (2013).

35. Budke, M., Allmers, T. \& Donath, M. Surface state vs orbital Kondo resonance at $\mathrm{Cr}(001)$ : arguments for a surface state interpretation. Phys. Rev. B 77, 233409 (2008)

36. Bode, M. et al. Chiral magnetic order at surfaces driven by inversion asymmetry. Nature 447, 190 (2007).

37. Trainer, C. et al. Manipulating surface magnetic order in iron telluride. Sci. Adv. 5, eaav3478 (2019)

38. Sacks, W., Roditchev, D. \& Klein, J. Voltage-dependent STM image of a charge density wave. Phys. Rev. B 57, 13118 (1998).

39. Mallet, P. et al. Contrast reversal of the charge density wave STM image in purple potassium molybdenum bronze $\mathrm{K}_{0.9} \mathrm{Mo}_{6} \mathrm{O}_{17}$. Phys. Rev. B 60, 2122 (1999).

40. Spera, M. et al. Insight into the charge density wave gap from contrast inversion in topographic STM images. Phys. Rev. Lett. 125, 267603 (2020)

41. Tsunoda, Y., Nakai, Y. \& Kunitomi, N. Phase relation between SDW and strain wave in chromium. Solid State Commun. 16, 443-445 (1975).

42. Mori, M. et al. Searching for charge density waves in chromium. J. Phys Condens. Matter 5, L77 (1993).

43. Machida, K. \& Fujita, M. Soliton lattice structure of incommensurate spindensity wolves: application to $\mathrm{Cr}$ and $\mathrm{Cr}$-rich $\mathrm{Cr}-\mathrm{Mn}$ and $\mathrm{Cr}-\mathrm{V}$ alloys. Phys. Rev. B 30, 5284 (1984).

44. Jiang, X. W. \& Fishman, R. S. Coupled spin- and charge-density waves in chromium alloys. J. Phys. Condens. Matter 9, 3417-3426 (1997).

45. Zhang, Y. et al. Competing orders in a magnetic field: Spin and charge order in the cuprate superconductors. Phys. Rev. B 66, 094501 (2002).

46. Balatsky, A. V., Basov, D. N. \& Zhu, J. X. Induction of charge density waves by spin density waves in iron-based superconductors. Phys. Rev. B 82, 144522 (2010).

\section{Acknowledgements}

We thank Prof. Chunlei Gao and Mr. Zhongjie Wang for the advice of preparing spinpolarized tip and helpful discussions. This work is supported by the National Key R\&D Program of the MOST of China with Grant Nos. 2017YFA0303004 (T.Z.), National Natural Science Foundation of China with Grant Nos. 92065202 (T.Z.), 11888101 (D.L.F.), 11790312 (D.L.F.), 11961160717 (T.Z.), Science Challenge Project with grant No. TZ2016004 (D.L.F.), Shanghai Municipal Science and Technology Major Project with Grant No. 2019SHZDZX01 (T.Z., D.L.F.), Science and Technology Commission of Shanghai Municipality, China (Grant Nos. 19JC1412702 (T.Z.), 21 TQ1400100 (T.Z.)).

\section{Author contributions}

The STM measurements and data analysis were performed by Y.N. Hu, T.Z. Zhang, D.M. Zhao, C. Chen, S.Y. Ding, W.T. Yang, X. Wang, C.H. Li, H.T. Wang and T. Zhang. D.L. Feng and T. Zhang coordinated the project and wrote the manuscript. All authors have discussed the results and the interpretation.

\section{Competing interests}

The authors declare no competing interests. 


\section{Additional information}

Supplementary information The online version contains supplementary material available at https://doi.org/10.1038/s41467-022-28104-2.

Correspondence and requests for materials should be addressed to Tong Zhang.

Peer review information Nature Communications thanks Erik van Heumen, Peter Wahl and the other, anonymous, reviewer(s) for their contribution to the peer review of this work. Peer reviewer reports are available.

Reprints and permission information is available at http://www.nature.com/reprints

Publisher's note Springer Nature remains neutral with regard to jurisdictional claims in published maps and institutional affiliations. (c) (P) Open Access This article is licensed under a Creative Commons Attribution 4.0 International License, which permits use, sharing, adaptation, distribution and reproduction in any medium or format, as long as you give appropriate credit to the original author(s) and the source, provide a link to the Creative Commons license, and indicate if changes were made. The images or other third party material in this article are included in the article's Creative Commons license, unless indicated otherwise in a credit line to the material. If material is not included in the article's Creative Commons license and your intended use is not permitted by statutory regulation or exceeds the permitted use, you will need to obtain permission directly from the copyright holder. To view a copy of this license, visit http://creativecommons.org/ licenses/by/4.0/.

(C) The Author(s) 2022 